\title{
Inhaltsverzeichnis Jahrgang 1911
}

Abhandlungen und Notizen.

Bork, F.: Die Kaspierkönige sind nicht arischer Herkunft .

- Zwei Dekanbildersysteme.

Brandenburg, (Mit 9 Abb. u. 3 Taf.).

Caspari, W.: Za Genesis 10......

Daiche日, S.: Dex Schmnek der Töehter Zions

Delaporte, L.: Da-da, patési de Nippour . .

Dittrich, E.: Za "Platons Zahlenrätsel"

Fries, C.: Phaiakenabentever und Lalitapistara.

Grimme, H. : Zu den aramäischen Achikarsprüchen

Gry, L.: Notes d'assyriologie

Gustars, A: Abd-hiba=Put-i-Hepa : .

Elartmann, M.: Kūga und Kügü . . . .

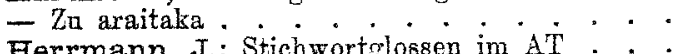

Herrmann, J.: Stichwortglossen in AT ${ }^{*}$.

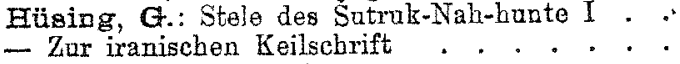

Jirku, A.: Das $b$ in beרrא Gen. 14... .

Jolles, A.: Etana oder Gilgameš .

Jurovics, S.: Stichwortglossen in AT . . .

Klauber, R.: Das Gartentor von Ninive . .

Künstlinger, D.: 'Zu D. H. Müllers Deutungen der hebräischen Buchstaben bei Ambrosius

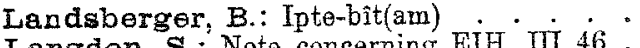

Langdon, S.: Note concerning FIH III 46 .

Mahler, E.: $0=$ Wrin,

Marmorstein, A.: Einige hebräische Redensarten Martin, F.: Les fonctions de l'uku-1uš . . .

Meissner, B.: aškapu

- Bemerkungen za den Asarhaddoninechriften

- Das Ende Suzubs

- Sebafschur in Babylonien.

Miller W M : Gosen nach einem demot. Schulbuch

- Die maghrebinische erste Person des Imperfelkts

Nestle, E.: Dio ersten hebräiseben Typon . .

- Zur Umschrift des Hebräischen . . . .

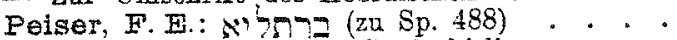

- Die neue Inschrift aus Sendschirli . . .

- Zu den Schutzrö̌celn ron Babylon . . : .

- Zum Ordal der Babylonier

Porles, F.: Splitter.

- Wer hat zuerst Strophen in der ATlichen Poesie angenommen? . . . . . . . . .

- Zu Sachaus "Aramäischen Papyrus"

Poebel, A.: Zur Aussprache der sumerischen Phrasen in den altbabylonischen Rechtsurkunden

- Zur Geierstele.

Schnabel, P.: Der Name der ersten Dynastie ron Babel bei Berossos . . . . . . . . . .

- Die Tomossummen des Manetho . . .

Schroeder, $0:$ mulmollu

Schultz, W.: Nachtrag zu Simsons Rätsel :

Seybold, C.'F.: Das arabisch-türkische Wörterbüehlein des Firište Oğlu $=$ Ibn (al) Malak

Spiegelberg, W.: Semit.Lehnwort im Demotischen

Strzygowski, J.: Problem d. persischen Kunst

Tallqvist, $\mathrm{K}$.: Das Datum des Feldzuges Sanheribs gegen Hilaku und des Eponymates Sulmu-bêls

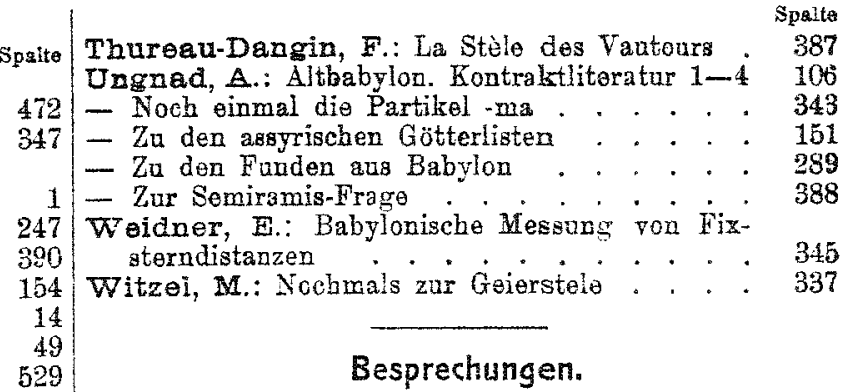

Thureau-Dangin, F.: La Stèle des Vautoura Spalte Uncnad. A.: Altbabylon. Kontraktliteratur $1-i$ i 100

201 - Noch einmal die Partikel -ma . . . . . . 343

347 - Zu den assyrischen Götterlisten . . . . . 151

- $\mathrm{Zu}$ den Funden aus Babylon . . . . . . 289

1 - Zur Semiramis-Frage . . . . . . . . . 388

247 Weidner, E.: Babylonische Messung von Fixsterndistanzen

WitzeI, M.: Nochmals zur Geierstele : : 337

14
49

Besprechungen.

sbel, H.: Zur Tonverschmelzung im Altägyptischen. (W. Wreszinski)

Albrecht, $\mathrm{K}$ : Register zur Zeitschrift für die atl. Wissenschaft. Bd. I-XXV, (F. B ork)

Armbruster, C. H.: Initia Amharica I, II. (E. Nittwoch)

Arno, T. J.: Sverigas Förbindelser med Oestem under vikingatiden. (C. Niobuhr) . . .

Bacher, W.: Die hebräische und arabische Poesio der Juden Jemens. (S. Poznański) - -

Basset, R.: La Banât Soâd. (M. Hartmann)

Bartels, W.: Die etruskische Bronzeleber ron Piacenza. (C. Fries) . . . . . . . . Eartholomae, Chr.: Ueber ein sasanidisches Rechtsbuch. (O. Mann) . . • $\cdot$.

Batiffol, P.: Orpheus et l'Évangile. (C. Fries) Baumbacher, J.: Im Banne von drei Föniginnen. (E. Brandenburg) , . . . ${ }^{\circ} \cdot{ }^{\circ}$ Baumstark, A.: Festbrevier und Kirchenjahr der sprischen Jakobiten. (E. Nestle) lépicraphie et l'histoire musulmanes du DiyarBeks:-J. Strzygowski, Zur Kunstgeschichte des Mittelalters von Nordmesopotamien, Hellas und dem Abendlande. (E. Herzfeld)

laufuss, H.: Götter, Bilder und Symbole nach denTraktaten über fremdenDienst.(W.Schultz)

Boeser, P. A. A.: Aegyptische Sammlung des Niederländischen Reichsmuseams in Leiden. (H. $\operatorname{Rank} \theta$ )

Boll. F.: Der griechische Kalender. (W. Schultz)

- Griech. Liebeszauber aus Aegypten. (C. Fries)

Brandt, W.: Jüd. Baptismen. (D. Künstlinger)

- Jidische Reinheitslabre. (D. Eitnstlinger) (J. Strzygowski)

Brockelmann, O.: s. Literaturen d. Ostens

Brunnhofer, E.: Buch der 100 Pfade. (H.Figulla)

Chabot, J. B.: l.es langues ot les littératures araméennes. (E. Nestle)

Chwolson, D.: Beiträga zur Entwicklnngsgeschichte des Judentums. (F. Perleg)

Ciccotti, E., Untargang der Sklaverei im Altertum. (C. Niebuhr)

Corpus Scriptorum Christianorum Orientaliam. Seript. Syri Zer. II. T. Cl. Dionysius bar Salibi in Apocalipsim ed. Sedlaček. (E. Nestle)

Crum, W. E.: Coptic manuscripts in the Jobn Rylands Library. (W. Spiagelborg) . 
Curipesobitz, B.: Itinerarium der Botschaftsreise des Josef yon Bamberg. (C. F. Seybold)

Delaporte, L.-J.: La Chronographie d'Élie barSinaya. (E. Nestle)

Dhorme, P. L religion assyro-babylonionne M. Pancritius) . . . . . . 110

Diehl, Ch.: Man.d'ArtByzantin.(E. Brandenburg)

Dietrich, B.: Kleinasiatischo Stickereien. (U. Brandonburgl

Dölger, F. J.: Der Exorcismus im altchristlichen Taufritual. (C. Fries).

Dörwald, P.: Der hebräische Unterricht. (F. Bork)

Dussaud, R.: Les civilisations préhelléniques, dans le basin de la Mer Éée. (E. Brandenburg)

Eisler, R.: Orpheus the fisher I, II, (C. Fries)

Eitrem, S.: Hermes und die Toten. (C. Fries)

Finck, F.N.: Haupttypea des Sprachbaus.(E.Lew y)

- s. Literaturen des Ostens

Fitzler, E.: Steinbrüche und Bergwerke im Ptole. mäischen u. Röraischen Aegypten. (C. Niebuhr)

Friedriohs, G.: Die Geschichtszahlen der Alten sind Kalenderzahlen. (W. Schultz)

Fries, O.: Studien zar Odysses I. (W.Schultz)

Fuchs, F.: Sagen, Mythen und Sitten der Masai. (W. Schultz)

Geiger, L. u. 2.: Abraham Geiger. (F. Perles)

Gibson, M. D. The commentaries of Isho'dad of Merw I, II, III (E. Nestle)

Girdlestone, R. B.:BibleCbronology. (Schaabel)

Graefe, B.: Das Pyramidenkapitel in al- Makrizzi's Hitat. (K. Lokotsch)

Graf, G.: Dio arabizehen Schriften des Theodor abu Qurra. (M. Horten)

Grossmann, H.: Palästinas Erdcreruch. (W. Erb t) - Altorientalische Texte und Bilder zum AT in Verbindg. mit A. Ungnad u.H.Ranke. (W. Erbt)

Grube, W.: Religion und Kultus der Chinesen. (A. Dekeley)

Grünert, M.: Arabische Lesestücke für Vorlesungszwecke H. 3. (H. Reckendorf).

B̈z̈nel, J.: Anssermasoreticche Uebereinstimmungen ZW. Septuaginta u. Peschittha. (Nestle).

Hahne, H.: D. vorgeschichtl. Europa. (F.E.Peiser)

Hamet, J.: Chroniques de la Mauritanio Sénégalaise. (M. Eartmann)

Harms, L.: Sebulwandkaxten. 4 a Palästina, $4 b$ Biblische Länder. (F. Bork)

Eassan b. Thäbit: Díwān, ed by H. Hirschfeld. (J. Barth)

Eerner, S.: Verbeaserungan zu Mandeikerns grosser Konkordanz. (J. Herrmann)

Herre, P,: Quellenkunde zur Weltgesch. (F. B ork)

Forrmann, A. Die alten Seidenstrassen zwischen Ohina and Syrien I (E. Brandenbarg)

Eerzfold. \#.: Genesis der islamisehen Kunst und das Msuatia Problem. (J. Strzygowski).

Hieratische Papyrus aus den Königlichen Museen zu Berlin 10. Heft, (A. Wiedemann) Hillobrandt, A.: Ved. Mythologie. (E.Si $\theta \mathrm{ck}$ ) 26 Horn, P.: s. Literaturen des Ostene

Jensen, P.: Moses, Jesus, Paulas. (W. Hrbt).

Jéquier, G: Décoration égrptienne I. (W. Wreszinski).

- Le papyrus Prisse et ses rariantes. (H. Ranke)

Junker, H.: Koptisehe Poesie des zelnten Jahrbunderts I, II. (W. Spiegelberg) . . .

Barutz, R.: Unter Kirgisen. (F. Bork)

Bing, I. W.: A history of Sumer and Akrad. (A. Ungnad)

König, E.: Hebrisches und aramöisches Wörterbuch zum AT. (J. Herrmann)

Kotelmann, L.: Vie Opbthalmologie bei den alten Hebräern. (J. Löw) . . . . .
Spalte

Künstlinger, D.: Das Achtzehngebet mit arsbischer Uebersetzung. (F. Perles) . . .

Lagrange, M.-J.: Quelques Remarques sur JOrpheus de M. S. Reinach. (C. Fries) .

Landersdorfer, S.: Die Bibel n. die aüdarabiache Altertumsforschung. (O. W eber)

121 Leipoldt, G.: s. Literaturen des Ostens

LeVY, A.: Die Syntax der koptischen Apophtheg. mata patrum Aegyptiorum. (W. Wreszinski)

v. Lioh tenberg, R.: Einflüsse der ägüischen Kultur auf Aegypten u. Palästina. (W. Wreszinski)

80 Tieblein 5: Recherches sur l'histoire de la civilisation de l'ancienne Égypte. (W. M. Müller)

86 Literaturen des Oatens Bd. VI, VII. (T. Mann)

218 Littmann, E.: s. Literaturen des Ostens.

Löschke, K.: Jüdisches und Heidnisches im christ-

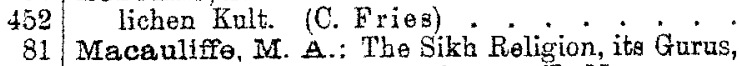
sacred writings aud Authors. (T. Mann)

221 Manassewitsch: Grammatik der hebräischen Sprache, 3. Aufl. v. B. Templer. (E. König)

303 Mann 0 : Rurdisch-persische Forschungen. Abt. I, II, IV. (E, Wilhelm) …

Margolis, G.: Lehrboch der aramäischen Sprache des babylonischen Talmuds. (J. Löw)

299 Massignon, L.: Mission en Mésopotamie (1907 bis 1908). (J. Strzygowski) . . . .

487 Moinh of, C.: Dio moderue Sprachforschung in

131 Afrika (TV. M Millor)

- Dir Sprache der Saabeli. (W. M. Müller)

522 - Lautlehre der Bantusprachen. (W. M. Müller)

Mélanges de la faculté orientalo de l'Université

128 de St. Joseph Beyr. II, III 1-2. (J. Horovitz) demischen Gebrauch H. 3. (H. Ranke) .

41 Munkácsi, B.: Sammlung wogulischer Volksdichtung II 2 . (R. Fuchs) . . . . . . .

322 Nikel, J.: Das alte Tostament im Lichte der altorientalischen Forschungen III. (J. Hehn)

129 Oppenheim, M. V.: Inschriften aus Syrien, Hesopot. u. Kleinasien I. (M. Sobernheim)

553 Palästinajahrbuch des Deutschen evangelischen Instituts in Jerusalem. (J. Herrmann).

Pfister, F.: Eleine Texte zum Alexanderroman. (C. Fries)

Pieper, M.: Brettspiel a a Aegrpter. (C. Fries)

166 Poebel, A. Personennamen. (A. Unguad)

จ. Reber, F.: Stellung der Hettiter in der Kunstgascuichte. Mrit 1 Abb. (F. Brandenburg)

Reinach. A.-J.: Rapport sur les Fouilles do Koptos, (WT. Wreszinski) , . . . .

37 Reinach, S.: Orpheus. (C. Fries). . . .

Röhl, K.: Versach einer systematischen Grammatik der Schambalasprache. (W. M. Mäller).

179 Roscher, W.: Die Tesserakontaden und Tesserakontadenlehren der Griechen u. a. Völker (u.) - Die Zabl 40 im Glauben, Erauch and Sehrift-

319 tum der Senniten. (C. Fries) . . . . . I II. (O). Mana)

212 I. II. (0. Sanders, E. A.: The Old Testamant Manuscripts in the Freer Collection. I. (iI. IJln $r$ )

38 Schormann.Th.: Griech.Zauberpapri. (C.Fries)

363 Schermann, Th, : Griech. Zauberpapyi. (F. Bork)

309 Schleifar, J.: Sabiaische Bibelfragmente ans dem

Britigh Nuseum, (W. Wreszinsti). .

Schmidt, W.: Die Mrthologio der austronesischen Yöker (u)

23 - Grundlinien e. Ferrieichung der Religionen u. Mrytlologien d. austronesischen Volker. (F. B or Z)

Schmitt, A.: Bibel a. Naturwissenschaft. (N.

479 Peters). . . . . . . . .
Spalte 
Schneider, H.: Żwei Aufsätze zur Religionsgeschichte Vorderasiens. (W. Erbt)

Schürer, F.: Geschichte des jüd. Volkes im Zeitalter J. Ch. 4. Aufl. Bd. III. (F. Perles) Schweinitz, H. H. $\nabla$. : Orientalische Wanderangen in Tarkestan. (O, Mann)

Sediaček, J.: s. Corpus Script. Christianorum . Slouschz, P.: Voyages d'étrades juives, en Afrique. (S. Poznański)

Sola Pool, D. de: The old Jewish-Aramaic Prayer the Kaddisb. (S. Poznański)

7. Spiess, K. : Prahistorie n.Mythos. (E. Siecke) Staerk, W.: Altjüdische liturgische Gebete, (v.) - Der Mišuatraktat Berakhot. (A Marx)

Stengel, P.: Opforbränche d. Griochen. (C. Fries) Strzygowki, J.: g. Berchen, Max yan . .

Sykes, B. C.: Persia and its people. (O. Mann)

Theis, J.: Geschiohtliche v. literarkritische Fragen in Esra J-6. (J. Hehn)

Theodorus bar Kōnĩ: Liber Scholioram, ed. A. Seher. (E. Nestle)

Thompson, H: A Coptic Palimpsest. (F. Rösch)

Thureau-Dangin, F.: Lettres et contrats de la prem. dynastie babylionenne. (B. Meissner)

Tolman, E. C.: Caneiform suppl. to the author's Persian Lexicon and Texts. (O. Mann) .

Trietsch, D.: Lerante Handbuch (n.)

- Marokko und Persien. (F. Bork)

Weyh, W: Die syrische Kosmas- and Damionlegende. (W. Schultz)

Wiedemann, M.: Bagdad u. Teheran. (Niebuhr)

WolfP, K. F': Die Germanen als Begründer der europäischen Kultur. (G. Hü ging)

Zeitsohrift f. Kolonialspr. I. 1, 2. (W. M. Müller)

Zimmern, E.: Zum Streit um die Christusmythe.

(M. Pancritius)

\section{Verzeichnis der Rezensenten.}

Barth, J. . . . 366

Bork, F. 37. 80.131. 166 $231.278,313,453$

Brandenbarg, E. 86. 121. 183. $279.435 .5+6$

Erbt, W. 78.212. 296. 44 L

Figulla, H. H. . 270 Fries, C. $35.36,117,174$.

218. 279. 314. 357.440

$$
484.522 .567
$$

Fuchs, R. . . 326

Hartmann, M. 129. 365

H $\odot$ hn, J. . . 449. 482

Herrmann, J. 259.451 .

483

Herzfeld, E. . 397

Horovitz, J. . . 32

Gortea, M. : . 128

Hüsing, G. . . .

Köuig, E. . . . 28

Künstlinger, D. $16 \dot{1} .359$

$L \in \mathbb{Y}, \mathrm{E} . \quad . \quad 452$

Löbr, M. . . 210

L. 0 w, J. : 127.479

Lokatseh, K. 529

Mann, O. 35. 82. 277. 325.

Mann, T. . 81. 272

Marx, A. 261

Meisaner, B. . . 21

Mittwocb, E. : : 71

Müler, W. M. $175,328$.

Sprechsaal.

Spalte-

\begin{tabular}{|c|c|}
\hline & Sprechsaal. \\
\hline & Bauer, L.: Zu OLZ 1910 Sp. 498 f. . . \\
\hline 20 & $\begin{array}{l}\text { Buchler, A.: Zn OAZ } 1911 \text { Sp. } 220 \text {. } \\
\text { Hartmann, MI: Makoraba. Eine Abwehr und }\end{array}$ \\
\hline 8 & 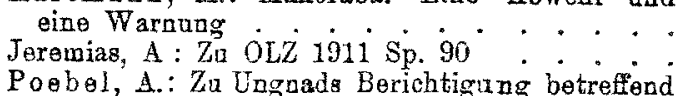 \\
\hline & $\begin{array}{l}\text { die Aussprache anmerischer Phrasen in alt- } \\
\text { babylonischen Rechtsurkunden } . .\end{array}$ \\
\hline & Sarsowsky, A.: Za OLZ 1911 Sp. 390 . \\
\hline & Onguad, A.: Zur Aussprache der sumerischen \\
\hline & $\begin{array}{l}\text { Phrasen in den altbabylonischen Her } \\
\text { kundon. Eino Bericbtigung . . . . }\end{array}$ \\
\hline
\end{tabular}

\section{Rus gelehrten Gesellschaften.}

Acad. des Inscriptions et B.-L. 39.133.235.330.374.492.56t

26 Société Asiatique, - Vorderasiatisch Gesellschaft 90

550 Institnt ethnographique international de Paris. . 185.

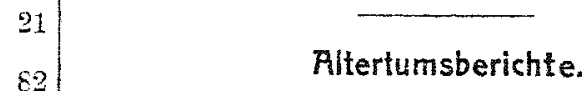

Erwerbungen der Berliner Museen 39. Grabungen in 278 Delos 39. Ausgrabungsfunde in Tiryns 132. - Ausgrabung eines dritten Tempels von Abydos 132. 118 Fund eines antiken Bronzelropfes im Hinterland von 524 Lome 133. - Leibgaben an die Islamigche Kunstabteilung der Berliner Museen 184

559
457

301

\section{$\mathbf{P}$}

Proisausschreiben des Lazarewschen Instituts in Moskn 40. - Aufnabme denotiseher Texte in Phylae durch W. M. Müller 90. - Ausgrabung in Sebastie (Samarien) 91. 133. 235. Ausgrabung von Samarra 185. - Orientalistenkongress in Athen 1912 186. - Compendium des Zeid ben Ali 186. - Ausgrabung in Samaria 235. - Stiftung de Goeje 235. - Congxès archéologiquo de France 282. - Congrès national des Sociétés franc. de Geographie 282. - Heransgaho einer petite bibliothèque arménienne 282 . - 49. Kongress der Sociétés savantes de Paris et des Départements 331 - Neve mubammedanische Unipersität 374. - Ausstellung chinesischer Kunst in Paris 374. - IV. Intern. Kongress für Religionsgeschichte 1912458 . - Nene Zeitschrift: Les musées de France 492. - 10. internat. Geographenkongress Rom 492. - Arabische Monateschrift: Loghatel:-Arab 492. - S. Guyer's Forschungsreise durch Wiedemann, A. . 519 Wilhelm, E. $\cdot 227$ Wreszinski, W 38.73.
259.311 .411 .489 Mesopotamien 493 . - Musenm in Konstantinopel 562.

\section{Personalien.}

Seybold, C. Siecke, E. 117.264 .316 Sobernheim, M. . 29 Spiegelberg,W. 257.309 Strzygowski, J. 179. 223 Uckeley, A. . . 322 Ungnad, A. . 21.223 Wober, O. . . 167

Reckendorf, H. . 129

Röseh, F. . . . 500

Schnabol, $\dot{P}:$ : 131

Schultz, W. 83. 118. 163. $303,350,437$

Berger, Élie 133. - Berger, Ph. 331. - Beylié, de 91. Uuyal, Rubens 493. - Eazuka, W. 564 . - Holl, J 4อ8. - Hilpresht, H. V. 31 . - Hoffmann, G 331. Jacob, G. 331. - Jung, J. 91. - Lieblein, J. 493. Mabler, Ed. 91. - Marquart. J. 458. 561. - Meissner, Br. 458. - Messersonmidt, Leop. 186. - Moritz, B. 374. - Naville, E. 37t. - Prohstein, Otto 186. Reich, E. 91. - Süssheim, K. 554. - Wilke, F. 91. 